\title{
Millikelvin cooling of the center-of- mass motion of a levitated nanoparticle
}

\section{Nathanaël P. Bullier, Antonio Pontin, Peter F. Barker}

Nathanaël P. Bullier, Antonio Pontin, Peter F. Barker, "Millikelvin cooling of the center-of-mass motion of a levitated nanoparticle," Proc. SPIE 10347, Optical Trapping and Optical Micromanipulation XIV, 103471K (6 September 2017); doi: $10.1117 / 12.2275678$

Event: SPIE Nanoscience + Engineering, 2017, San Diego, California, United States 


\title{
Millikelvin Cooling of the Center-of-Mass Motion of a Levitated Nanoparticle
}

\author{
Nathanaël P. Bullier, Antonio Pontin, and Peter F. Barker \\ Department of Physics and Astronomy, University College London, Gower Street, London, \\ WC1E 6BT, United-Kingdom
}

\begin{abstract}
Cavity optomechanics has been used to cool the center-of-mass motion of levitated nanospheres to millikelvin temperatures. ${ }^{1}$ Trapping the particle in the cavity field enables high mechanical frequencies ${ }^{1-3}$ bringing the system close to the resolved-sideband regime. Here we describe a Paul trap constructed from a printed circuit board that is small enough to fit inside the optical cavity and which should enable an accurate positioning of the particle inside the cavity field. This will increase the optical damping and therefore reduce the final temperature by at least one order of magnitude. Simulations of the potential inside the trap enable us to estimate the chargeto-mass ratio of trapped particles by measuring the secular frequencies as a function of the trap parameters. Lastly, we show the importance of reducing laser noise to reach lower temperatures and higher sensitivity in the phase-sensitive readout.
\end{abstract}

Keywords: cavity optomechanics, cavity cooling, Paul trap, levitated nanosphere, frequency noise

\section{INTRODUCTION}

Many recent experiments have brought optomechanics one step closer to test quantum mechanics at nanoscales and probe nonclassical motions. These breakthroughs include cooling oscillators to their quantum ground state, ${ }^{4}$ performing quantum non-demolition measurements ${ }^{5}$ and measuring squeezed states of the mechanical oscillator. ${ }^{6}$ Levitated optomechanics has the advantage over clamped systems of providing greater thermal isolation giving higher mechanical Q factors so that longer coherence times could be achievable. Furthermore, the possibility to quickly reshape the trapping potential, or completely shutting it off, can allow for more advanced protocols. ${ }^{7}$ Finally, it provides a higher frequency tunability. ${ }^{8}$ Cooling the center-of-mass motion of levitated nanospheres has several motivations ranging from foundations of quantum mechanics to ultra-precise sensing. Indeed, such systems could play a key-role in obtaining a better understanding of the quantum/classical frontier. The advantages, cited above, over clamped systems make them very promising systems to show evidence of quantum tunneling ${ }^{9}$ and mechanical Fock states. ${ }^{10}$ Probing nonclassical behaviors using these systems allows us to explore quantum decoherence and potentially wave-function collapse models. ${ }^{7}{ }^{11}$ Lastly, levitated optomechanics has potential applications in measuring very small forces which is relevant for gravitational-wave detections ${ }^{12}$ as well as Casimir forces or non-Newtonian gravity. ${ }^{13}$

\section{COOLING MECHANISM}

So far two main cooling mechanisms have been used to cool the center-of-mass motion of levitated nanospheres. One is feedback cooling which applies a velocity proportional force to the particle either in the 3D cold damping scheme ${ }^{14}$ or using a non-linear parametric scheme. ${ }^{15}$ The other approach is cavity cooling which relies on the dipole interaction between a levitated nanosphere and the optical field. Both have been proven to be effective, however, here we focus on the second approach where the cavity field acts on the momentum of the sphere via the gradient force, and the position dependent polarization detunes the cavity from resonance. By red-detuning the input field with respect to the optical resonance the cavity back-action leads to cooling of the mechanical

Further author information: (Send correspondence to P.F.B.)

P.F.B.: E-mail: p.barker@ucl.ac.uk

Optical Trapping and Optical Micromanipulation XIV, edited by Kishan Dholakia, Gabriel C. Spalding, Proc. of SPIE Vol. 10347, 103471K · @ 2017 SPIE · CCC code: 0277-786X/17/\$18 · doi: 10.1117/12.2275678 
motion. ${ }^{16}$ The particle needs to be on the side of the antinode at $\lambda / 8$ for maximum cooling. This can be achieved with a Paul trap.

The hybrid trap profile can be seen in Fig. 1. It consists of the overlap between the cavity optical standing wave and the Paul trap potential. The oscillating Paul trap potential at the drive frequency $\omega_{d}$ results in the micromotion of charged nanoparticles. As a consequence the particle moves away from the antinode to regions where the linear coupling is non vanishing. This micromotion depends on trap and particle parameters. Its typical timescale is much slower than the dynamics in the optical potential which allows to separate the two evolutions. If the particle is trapped in the center of the Paul trap, that is to say in the well $\mathrm{N}=1$, it does not get any cooling from the micromotion (see Fig. 1). The averaged linear optomechanical coupling strength over one micromotion period $\bar{G}$ can be shown to be proportional to the number of charges, the Paul trap drive voltage and the well number N. This gives rise to an averaged cooling rate $\Gamma_{o p t}=\bar{G}^{2} \kappa\left(S\left(\omega_{m}\right)-S\left(-\omega_{m}\right)\right)$ with $\kappa$ the cavity decay rate and $S$ the optical susceptibility.

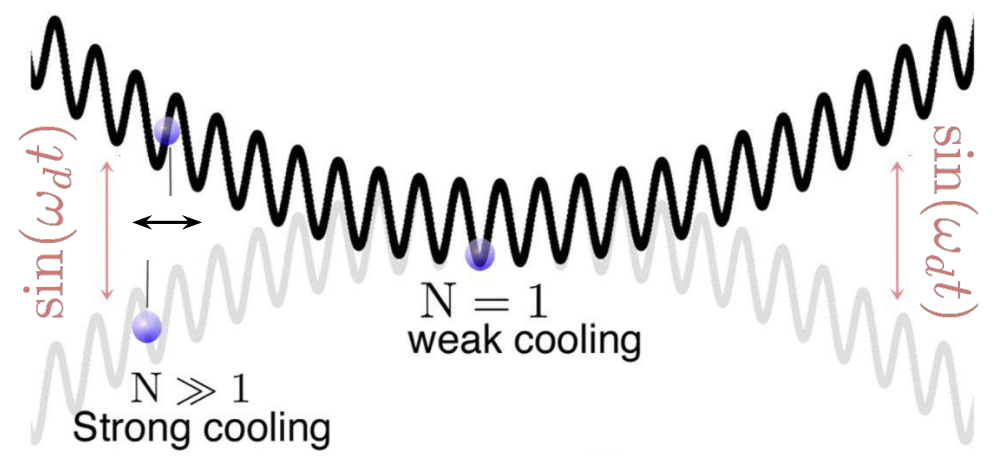

Figure 1. Particle in the hybrid electro-optical potential. The particle-light coupling is highly nonlinear and both linear and quadratic couplings have been reported. ${ }^{1}$ The particle micromotion in the Paul trap at the drive frequency $\omega_{d}$ leads to some excursion of the particle away from the antinode where there is a non vanishing linear optomechanical coupling. The amplitude of the micromotion is proportional to the Paul trap voltage, the number of charges on the particle and the well number N. Figure adapted from Ref. 17 with kind permission of the authors.

Cavity cooling of a nanoparticle in the hybrid trap has been demonstrated. ${ }^{1,3}$ There are a number of important improvements that are required to reach the ground state. These include improvements in the Paul trap as well as the reduction of the major noise sources. In this paper we describe the construction of a new Paul trap which allows us to precisely control the position of the particle in the cavity field to maximize the cooling. We also present initial measurements of laser intensity noise and frequency noise and show how these affect the cooling and the sensitivity of the measurement readout.

\section{PAUL TRAP DESIGN AND SIMULATIONS}

A standard linear Paul trap generally consists of four rods on which an AC voltage is applied to confine charged ions or spheres in the orthogonal plane to the rods. ${ }^{18}$ The nanospheres can be confined in the direction of the rods by applying DC voltages on so-called end-caps. We show here a PCB (printed circuit board) design which has the advantage of fitting inside our optical cavity. In the PCB based Paul trap (see Fig. 2), the copper tracks constitute the rods. By attaching two printed boards on top of each other, one can form a Paul trap (see Fig. 2.C). The space left between the two PCB can be used for optical manipulations as shown in Fig. 2.C. The hole above and below the center of the trap is used for both imaging and injecting particles into the trap.

In Fig. 2.A and Fig. 2.B are shown two different models of the trap. The former has four end-caps whereas the latter has height. The former gives the possibility to move the center of the trap in the $\mathrm{x}-\mathrm{z}$ plane whereas the latter enables to move it in the three dimensions. 

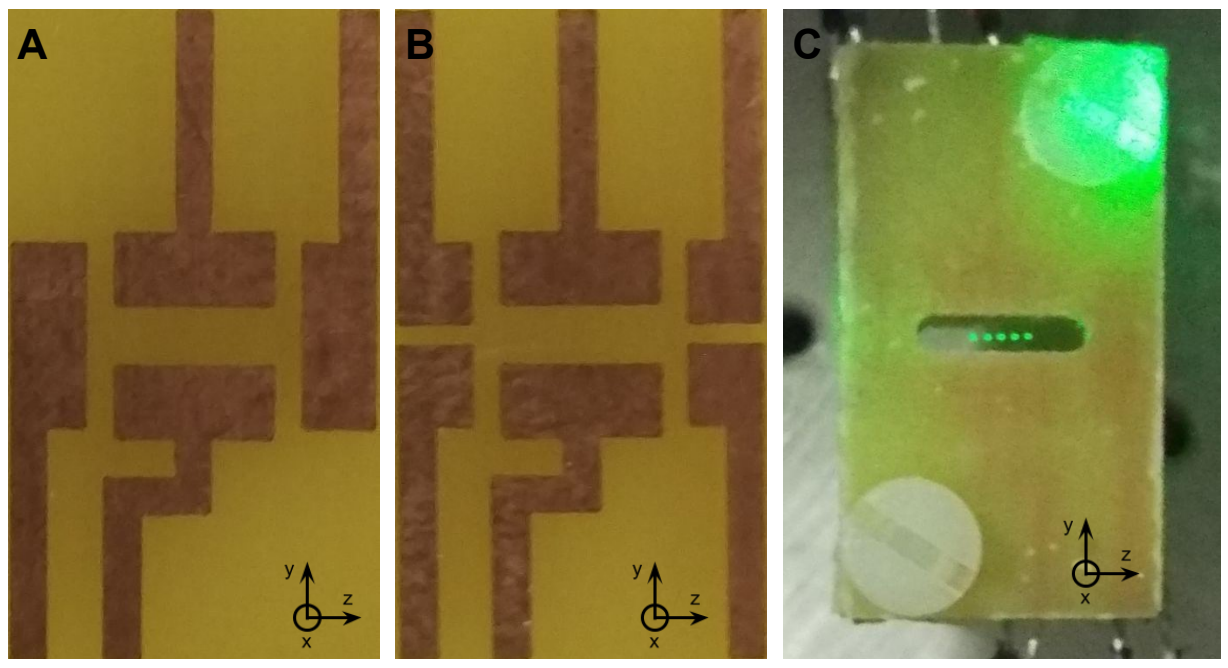

Figure 2. Fig.2.A and Fig.2.B are pictures of different circuits used for the Paul trap. In Fig.2.A the two elongated electrodes in the z-direction are the AC ones. The two elongated ones in the y-direction are the end-caps used to confine nanospheres in the z-direction. In Fig.2.B. one can see a design with four end-cap electrodes which gives a 3D control over the position of the center of the trap. In Fig.2.C is shown the Paul trap with trapped particles. The trap is assembled by fixing two printed circuits on top of each other. The two PCB are separated by $1.4 \mathrm{~mm}$. The copper layers have a thickness of $70 \mu \mathrm{m}$. The distance between the two AC electrodes is $1.6 \mathrm{~mm}$ while the distance between the end-caps is $5.8 \mathrm{~mm}$.

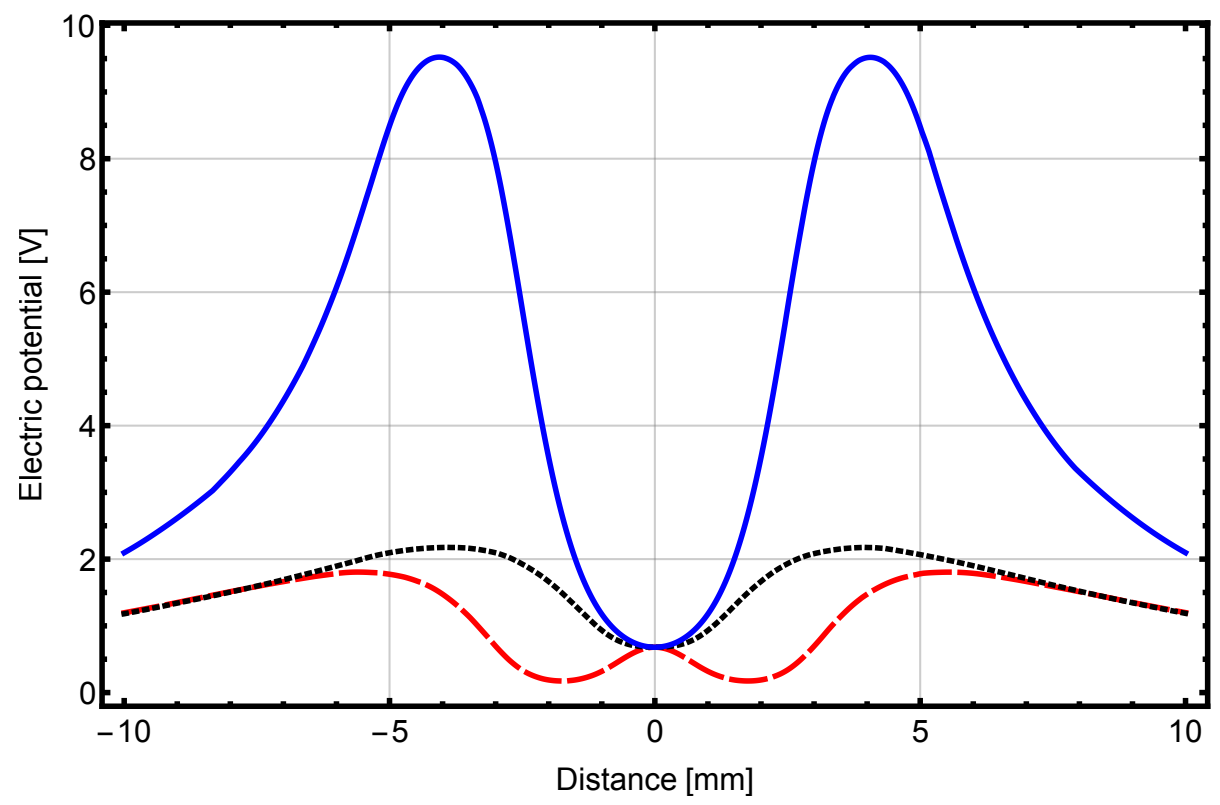

Figure 3. Simulation showing the electric potential in the trap given by the end-caps (with AC electrodes grounded). A typical value of $10 \mathrm{~V}$ is applied on the end-caps. The red dashed line corresponds to the $\mathrm{x}$-axis, the black dotted line to the y-axis and the solid blue line to the z-axis. The three axis correspond to the three directions shown in Fig. 2. The trapping potential is the strongest in the z-direction as expected. To be noticed the anti-trapping in the $\mathrm{x}$-direction. 


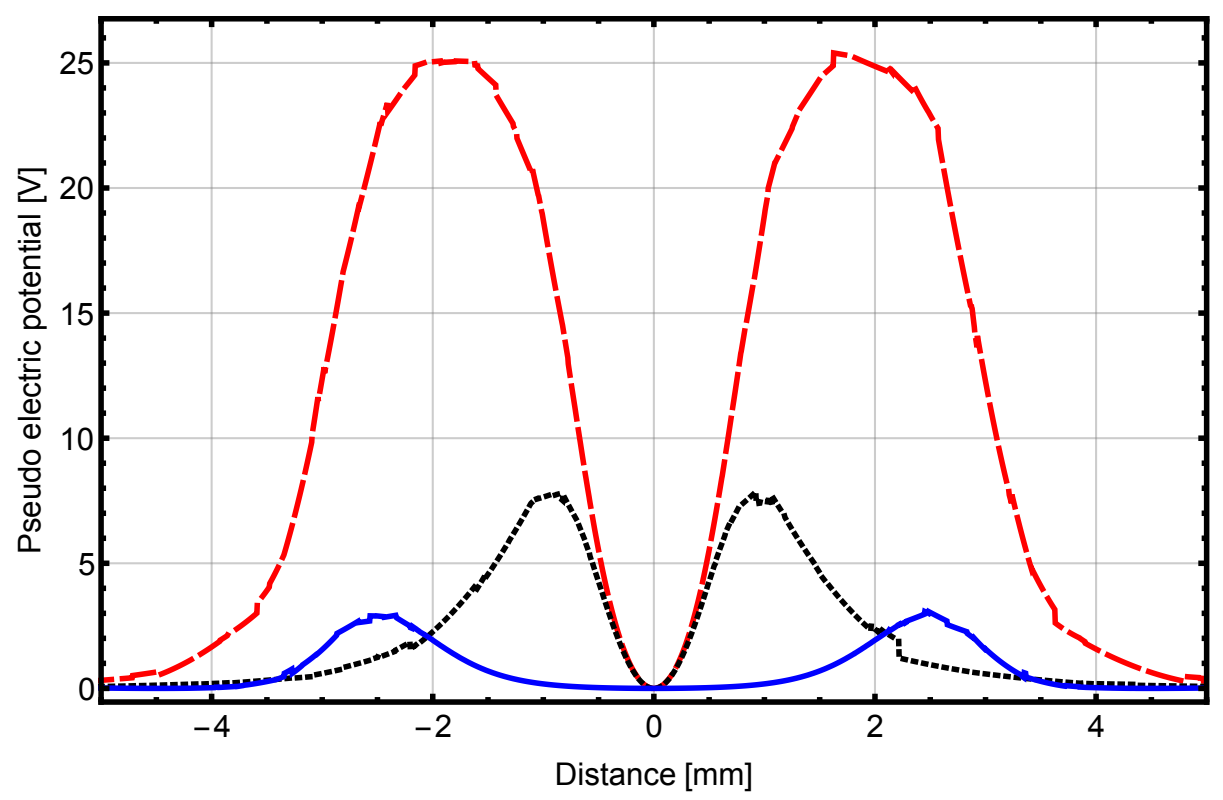

Figure 4. Simulation showing the pseudo electric potential in the trap given by the AC electrodes (with end-cap electrodes grounded). The red dashed line corresponds to the x-axis, the black dotted line to the y-axis and the solid blue line to the z-axis. The three axis correspond to the three directions shown in Fig. 2. This pseudo electric potential was obtained from a DC simulation and scaled accordingly with typical parameters. A voltage amplitude of $500 \mathrm{~V}$ was considered with a particle charge-to-mass ratio of $0.1 \mathrm{C} / \mathrm{kg}$ and a drive frequency of $1 \mathrm{kHz}$. To be noticed the residual trapping potential in the z-direction.

Following from Ref. 19 simulations of the potential across the trap were carried out using a finite-element software to get a simple theoretical model of the secular frequencies as a function of trap parameters and chargeto-mass ratio. The PCB design used for those simulations is shown in Fig. 2.A. Two static simulations are required to characterize the overall trap potential given by the superposition of the DC potential generated by the end-caps and the $\mathrm{AC}$ pseudo-potential. In the former case, a given potential $U_{o}$ is applied to the four end-caps while the other electrodes are grounded. In the latter, the end-caps are grounded and a given potential $V_{o}$ is applied on two opposing electrodes while the other two are grounded. In both cases the result of the simulation is normalized to the applied voltage. While the end-caps potential directly provides the trap depth, the static simulation on the AC electrodes allows to calculate it through the pseudo-potential $\frac{q V_{0}^{2}}{4 m \omega_{d}}|\nabla V(x, y, z)|^{2}$ where $V(x, y, z)$ is the normalized simulated potential, $q / m$ the charge-to-mass ratio and $\omega_{d}$ the drive frequency. We show in Fig. 3 and Fig. 4 the expected trapping potential corresponding to the potential created by the end-caps and the AC rods respectively. To allow a more direct comparison we consider a set of parameters used experimentally. For the end-caps we have a potential $U_{o}=10 \mathrm{~V}$. As expected the electric field gives rise to a strong potential well in the z-direction. In the y-axis the resulting potential is trapping while anti-trapping in the x-direction in agreement with Earnshaw's theorem. For the pseudo-potential, we consider a drive voltage $V_{o}=500 \mathrm{~V}$, drive frequency $\omega_{d} / 2 \pi=1 \mathrm{kHz}$ and a charge-to-mass ratio of $0.1 \mathrm{C} / \mathrm{kg}$. The potential is the strongest in the $\mathrm{x}$-direction while weaker in the $\mathrm{y}$-direction because of the electrodes being closer to each other in the $\mathrm{x}$-direction. To be noticed the trapping potential in the $\mathrm{z}$-direction coming from the fact that the electrodes are finite in size.

The final trapping potential consists of the sum of the DC potential and the AC pseudo-potential. By comparing the field near the center of the trap to a perfect quadrupole one, one can extract geometric and efficiency parameters. ${ }^{19}$ This gives rise in our case to theoretical expressions of the secular frequencies

$$
\omega_{x}=\sqrt{\frac{q^{2}}{m^{2}} \frac{V_{o}^{2} \eta^{2}}{2 l^{4} \omega_{d}^{2}}-\epsilon \frac{q}{m} \frac{2 U_{o} \kappa}{d^{2}}},
$$




$$
\begin{aligned}
\omega_{y} & =\sqrt{\frac{q^{2}}{m^{2}} \frac{V_{o}^{2} \eta^{2}}{2 l^{4} \omega_{d}^{2}}-(1-\epsilon) \frac{q}{m} \frac{2 U_{o} \kappa}{d^{2}}}, \\
\omega_{z} & =\sqrt{\frac{q^{2}}{m^{2}} \frac{V_{o}^{2} \eta^{2} \sigma^{2}}{2 l^{4} \omega_{d}^{2}}+2 \frac{q}{m} \frac{2 U_{o} \kappa}{d^{2}}}
\end{aligned}
$$

where $\omega_{x}, \omega_{y}$ and $\omega_{z}$ correspond to the secular frequencies in the $x, y$ and $z$ directions respectively. $q$ is the particle charge, $m$ the particle mass. The geometric parameters are $l$ and $d$, distance between the center of the trap and the nearest point of the $\mathrm{AC}$ electrodes and $d$ distance between the center of the trap and the nearest point of the end-caps respectively. The drive has an amplitude peak-to-peak $V_{o}$ and a frequency $\omega_{d}$. The DC voltage applied on the end-caps is $U_{o}$. The geometric efficiencies obtained after comparing the potentials to pure quadrupole ones are $\eta^{*}, \epsilon, \sigma$ and $\kappa$. It is worth noting that unlike traditional quadrupole traps, the frequency degeneracy in the $\mathrm{x}$ and $\mathrm{y}$ directions is lifted by the end-caps potential. Furthermore, depending on the value of $\epsilon$, the potential given by the end-caps in the y-axis can be trapping or anti-trapping. The values of the Paul trap parameters can be found in Table 1.

Table 1. Paul trap parameters

\begin{tabular}{lcccccc}
\hline symbol & $l(\mathrm{~mm})$ & $d(\mathrm{~mm})$ & $\eta$ & $\sigma$ & $\epsilon$ & $\kappa$ \\
\hline value & 1.083 & 2.991 & 0.349 & $6.18 \times 10^{-3}$ & 1.31 & 0.382 \\
\hline
\end{tabular}

\section{MEASURING THE CHARGE-TO-MASS RATIO}

The charge-to-mass ratio can be calculated by measuring the secular frequencies and comparing them to the model given above. The three secular frequencies can be measured as a function of the three trap parameters: AC drive amplitude and frequency, and DC voltage applied on the end-caps. By fitting the secular frequencies one can estimate the charge-to-mass ratio. The secular frequencies were measured with a tightly focused (waist of 14 $\mu \mathrm{m}$ ) infrared laser beam on a one micron (diameter) nanosphere. Interferences between the transmitted and the scattered field were measured using a quadrant photodiode. The particles were injected at atmospheric pressure with a nebulizer. Measurements were carried out in vacuum at pressures between $10^{-1}$ mbar and $10^{-3}$ mbar. A few examples for a same particle are shown in Fig. 5. The charge-to-mass ratio can be fitted and one gets from these three measurements $0.23,0.45$ and $0.17 \mathrm{C} / \mathrm{kg}$ respectively. Discrepancies in the measurements can be explained by the fact that the charge-to-mass ratio was the only free parameter in the fits as well as imprecision in the fabrication and assembly. Once the charge-to-mass ratio is calculated, the number of charges can be determined from the mass of the particle. The particle size can be measured with a linewidth measurement.
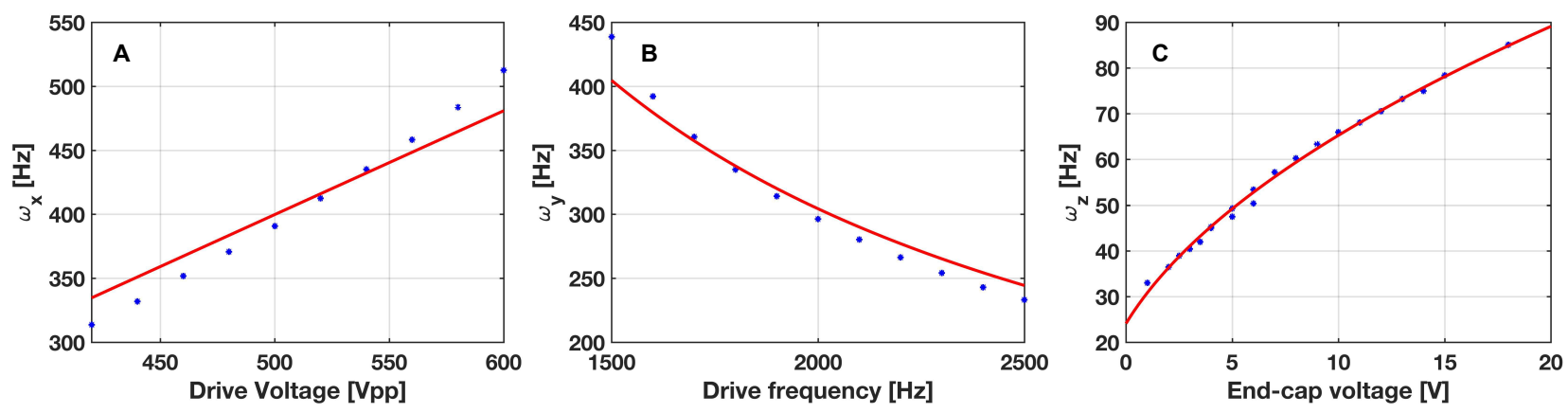

Figure 5. In $\mathrm{A}$ is shown the secular frequency in the $\mathrm{x}$-direction as a function of the drive voltage. In $\mathrm{B}$ is shown the secular frequency in the $\mathrm{y}$-direction as a function of the drive frequency. In $\mathrm{C}$ is shown the secular frequency in the $\mathrm{z}-$ direction as a function of the end-cap voltage. The fit gives a charge-to-mass ratio of $0.23,0.45$ and $0.17 \mathrm{C} / \mathrm{kg}$ respectively. This corresponds to an estimated number of charges of 1700, 3200 and 1200.

\footnotetext{
${ }^{*}$ The value of $\eta$ is doubled when $\mathrm{AC}$ voltages are applied on the four electrodes
} 


\section{LASER CLASSICAL NOISE}

Nanospheres trapped by the cavity field have mechanical frequencies in the $100 \mathrm{kHz}$ range. At these frequencies commercial lasers are not shot-noise limited which can result in both heating and limitations in the signal-tonoise ratio of a phase-sensitive measurement. Here we show with a toy-model the effects of laser noise on our experiment. The noise sources considered are the frequency noise and intensity noise. In this model heating of the particle due to cavity displacement noise, recoil losses, black-body radiation and Paul trap noise are neglected. Optical losses in the detection are neglected. Furthermore, the dynamics are linearized and the Paul trap micromotion is only taken into account in the averaged value of the optical damping over one micromotion period (see SI from Ref.1). This neglects split-sideband dynamics fully treated in Ref. 20. We consider silica spheres of $400 \mathrm{~nm}$ (diameter) with 3 charges. The particle is assumed to be trapped in the well number $\mathrm{N}=350$. The input beam has a power of $12 \mathrm{~mW}$ and is red-detuned by $100 \mathrm{kHz}$ which gives rise to a mechanical frequency of $100 \mathrm{kHz}$. A double-sided cavity with same transmission coefficients of $5 \mathrm{ppm}$ is considered. The cavity finesse is 200000 with a length of $13 \mathrm{~mm}$. The system is at $300 \mathrm{~K}$ and is operated at $5 \times 10^{-4}$ mbar. Our laser intensity noise at $100 \mathrm{kHz}$ is measured to be $3 \mathrm{~dB}$ above shot noise at $35 \mu \mathrm{W}$. The frequency noise at $100 \mathrm{kHz}$ is 0.04 $\mathrm{Hz}^{2} / \mathrm{Hz}$ experimentally measured with a delay line Michelson interferometer. The frequency noise is incorporated into the model as an effective cavity detuning.

In Fig. 6 one can see the contribution to heating from the different sources of noise considered: thermal noise, laser frequency and intensity noise, and radiation pressure shot noise. At this pressure, the thermal heating and the frequency noise have very close contribution. In Fig. 7 is shown the contribution of the different sources of noise in the homodyne detection of the reflected field. The frequency noise sets up a very high noise floor which reduces the signal-to-noise ratio, making it difficult to resolve the motion of the particle at lower pressures. These classical laser noise sources can both be reduced by implementing a filtering cavity which we plan in future work.

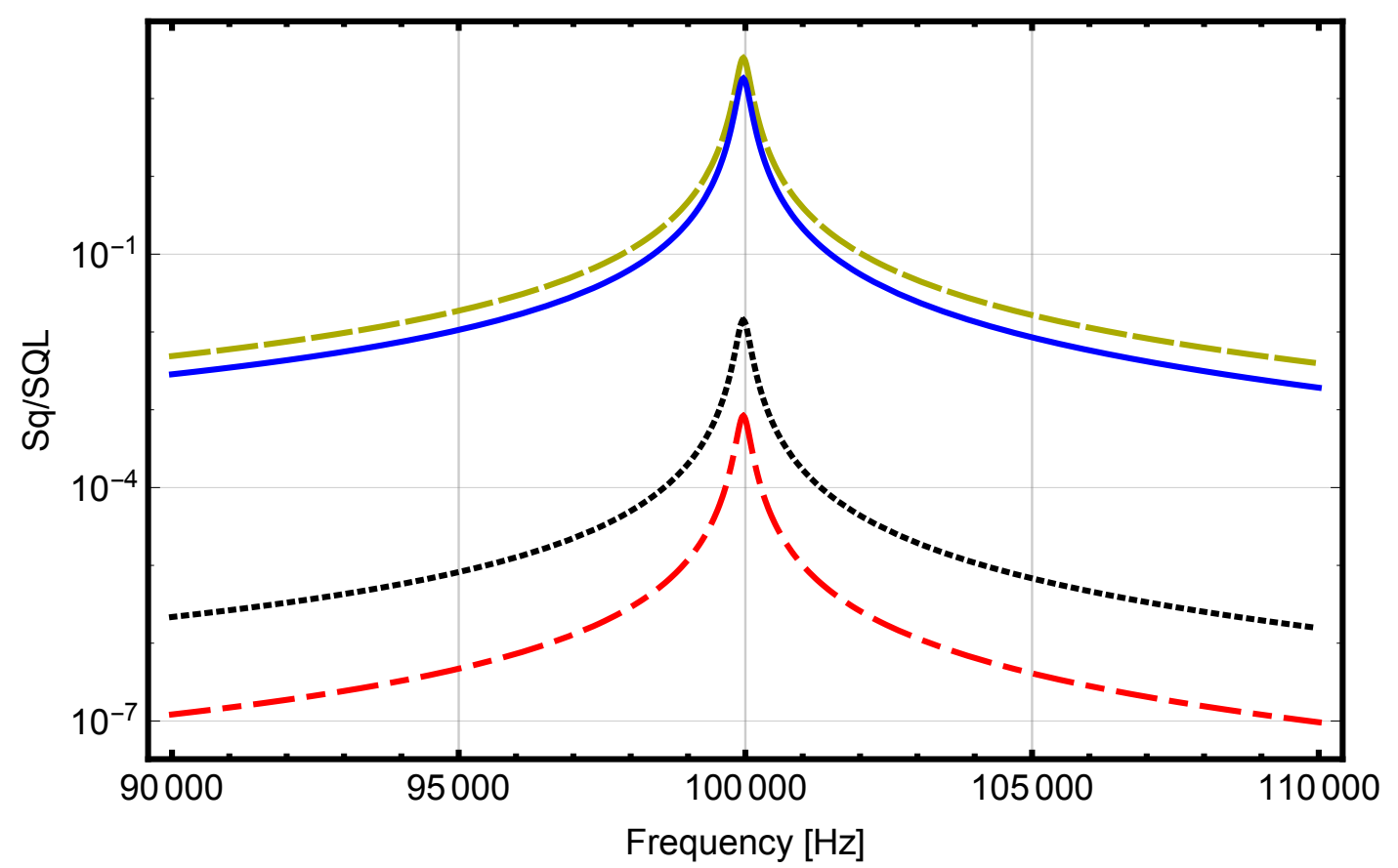

Figure 6. Contribution from the different sources of noise to the position spectrum of the particle at $5 \times 10^{-4} \mathrm{mbar}$. The blue solid line corresponds to the laser frequency noise, the black dotted one to the laser intensity noise, the yellow one with long dashes to the thermal noise and the dot-dashed red one to the radiation pressure shot noise. The spectrum is normalized to the zero-point-motion of the nanosphere. At this pressure both thermal noise and laser frequency noise have comparable contributions. The contributions in terms of phonon number from the thermal noise to the radiation pressure are 24000, 13000, 10 and 0.6 respectively. 


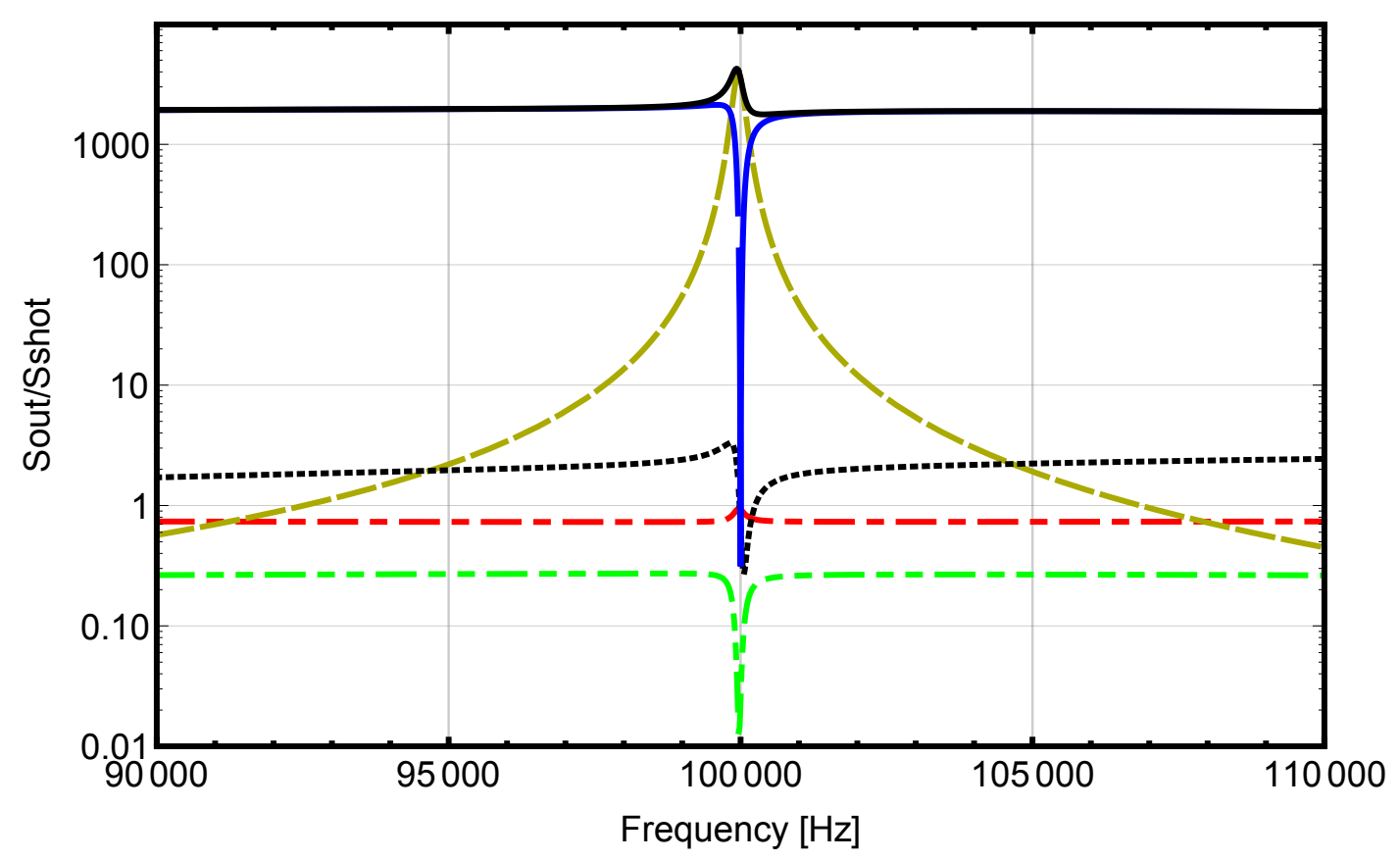

Figure 7. Contribution from the different sources of noise to the phase quadrature of the homodyne spectrum of the reflected cavity mode at $5 \times 10^{-4}$ mbar. The blue solid line corresponds to the laser frequency noise, the black dotted one to the laser intensity noise, the yellow one with long dashes to the thermal noise, the dot-dashed red one to the input shot noise, the double-dot-dashed green one to the vacuum losses and the black solid line to the total. The spectrum is normalized to the photon shot-noise. The frequency noise sets-up a very high noise floor limiting the sensitivity of the detection at lower pressures.

\section{CONCLUSION}

To conclude we have demonstrated a small linear Paul trap built on a printed circuit board. The fine control given by the end-caps on the position of the center of the Paul trap can be used to control the position of the particle in the cavity field. This could be used to maximize the optical damping and therefore lower the final temperature of the particle. We have derived a model that has enabled us to estimate the number of charges on the particle. Lastly, we have shown the importance of reducing laser classical noise in the system to reduce heating and improve the sensitivity of the measurement readout.

\section{ACKNOWLEDGMENTS}

The authors thank Anas Almuqhim for helping them designing the Paul trap. The authors acknowledge funding from the EPSRC Grant No. EP/N031105/1. N.P.B. acknowledges funding from the EPSRC Grant No. EP/L015242/1. This project has received funding from the European Unions Horizon 2020 research and innovation programme under the Marie Sklodowska-Curie Grant Agreement No. 749709.

\section{REFERENCES}

[1] Fonseca, P. Z. G., Aranas, E. B., Millen, J., Monteiro, T. S., and Barker, P. F., "Nonlinear dynamics and strong cavity cooling of levitated nanoparticles," Phys. Rev. Lett. 117, 173602 (Oct 2016).

[2] Kiesel, N., Blaser, F., Deli, U., Grass, D., Kaltenbaek, R., and Aspelmeyer, M., "Cavity cooling of an optically levitated submicron particle," Proceedings of the National Academy of Sciences 110(35), 1418014185 (2013).

[3] Millen, J., Fonseca, P. Z. G., Mavrogordatos, T., Monteiro, T. S., and Barker, P. F., "Cavity cooling a single charged levitated nanosphere," Phys. Rev. Lett. 114, 123602 (Mar 2015). 
[4] Chan, J., Alegre, T. P. M., Safavi-Naeini, A. H., Hill, J. T., Krause, A., Groblacher, S., Aspelmeyer, M., and Painter, O., "Laser cooling of a nanomechanical oscillator into its quantum ground state," Nature $\mathbf{4 7 8}$, 89-92 (10 2011).

[5] Suh, J., Weinstein, A. J., Lei, C. U., Wollman, E. E., Steinke, S. K., Meystre, P., Clerk, A. A., and Schwab, K. C., "Mechanically detecting and avoiding the quantum fluctuations of a microwave field," Science 344(6189), 1262-1265 (2014).

[6] Wollman, E. E., Lei, C. U., Weinstein, A. J., Suh, J., Kronwald, A., Marquardt, F., Clerk, A. A., and Schwab, K. C., "Quantum squeezing of motion in a mechanical resonator," Science 349(6251), 952-955 (2015).

[7] Goldwater, D., Paternostro, M., and Barker, P. F., "Testing wave-function-collapse models using parametric heating of a trapped nanosphere," Phys. Rev. A 94, 010104 (Jul 2016).

[8] Chang, D. E., Regal, C. A., Papp, S. B., Wilson, D. J., Ye, J., Painter, O., Kimble, H. J., and Zoller, P., "Cavity opto-mechanics using an optically levitated nanosphere," Proceedings of the National Academy of Sciences 107(3), 1005-1010 (2010).

[9] Buchmann, L. F., Zhang, L., Chiruvelli, A., and Meystre, P., "Macroscopic tunneling of a membrane in an optomechanical double-well potential," Phys. Rev. Lett. 108, 210403 (May 2012).

[10] Rips, S., Kiffner, M., Wilson-Rae, I., and Hartmann, M. J., "Steady-state negative wigner functions of nonlinear nanomechanical oscillators," New Journal of Physics 14(2), 023042 (2012).

[11] Bassi, A., Lochan, K., Satin, S., Singh, T. P., and Ulbricht, H., "Models of wave-function collapse, underlying theories, and experimental tests," Rev. Mod. Phys. 85, 471-527 (Apr 2013).

[12] Arvanitaki, A. and Geraci, A. A., "Detecting high-frequency gravitational waves with optically levitated sensors," Phys. Rev. Lett. 110, 071105 (Feb 2013).

[13] Geraci, A. A., Papp, S. B., and Kitching, J., "Short-range force detection using optically cooled levitated microspheres," Phys. Rev. Lett. 105, 101101 (Aug 2010).

[14] Li, T., Kheifets, S., and Raizen, M. G., "Millikelvin cooling of an optically trapped microsphere in vacuum," Nat Phys 7, 527-530 (07 2011).

[15] Gieseler, J., Deutsch, B., Quidant, R., and Novotny, L., "Subkelvin parametric feedback cooling of a lasertrapped nanoparticle," Phys. Rev. Lett. 109, 103603 (Sep 2012).

[16] Kippenberg, T. J. and Vahala, K. J., "Cavity optomechanics: Back-action at the mesoscale," Science 321(5893), 1172-1176 (2008).

[17] Aranas, E. B., Fonseca, P. Z. G., Barker, P. F., and Monteiro, T. S., "Thermometry of levitated nanoparticles in a hybrid electro-optical trap," Journal of Optics 19(3), 034003 (2017).

[18] March, R. E., "An introduction to quadrupole ion trap mass spectrometry," Journal of Mass Spectrometry 32(4), 351-369 (1997).

[19] Madsen, M., Hensinger, W., Stick, D., Rabchuk, J., and Monroe, C., "Planar ion trap geometry for microfabrication," Applied Physics B 78, 639-651 (Mar 2004).

[20] Aranas, E. B., Fonseca, P. Z. G., Barker, P. F., and Monteiro, T. S., "Split-sideband spectroscopy in slowly modulated optomechanics," New Journal of Physics 18(11), 113021 (2016). 